\title{
IoT Based Vehicle for Industrial Environment Monitoring
}

\author{
D. Usha Nandini, S. Prince Mary, R. Sathyabama Krishna, B. Bharathi, V. Maria Anu
}

\begin{abstract}
Industrial establishments always involve risk to the environment and people living there. Statistical analysis of the major-accidents occurred in the last few decades indicate that the reason for the industrial accidents is either 'human errors' or failure of the machines.So, there is need to analyse the consequences of potential accidents for the purpose of emergency planning and disaster management. This proposed vehicle is equipped with various gas sensors(specificto the industry) which is capable of detecting gases and alerts the admin using GSM module and it also stores real time status of the environmentin cloud through the use of IoT module andthe updates are notified to the admin.
\end{abstract}

Index Terms-Gas Detection, Industrial Automation, Pollution monitoring, Obstacle avoidance.

\section{INTRODUCTION}

Internet of things (IoT) is not a new technology it is around 16 years old. But the idea of connecting devices is much older than IoT itself, it has been around us at least since the past three decades .But this concept was known to people as "embedded internet" or "pervasive computer". The term "Internet of Things" was coined by Kevin Ashton in 1999 $[1,10]$. The concept of IoT gained popularity around the year 2010 and since then various applications of IoT have been developed for its use in different platforms.

IoT has played a big role in Industrial Automation too. There are various instances where IoT is being applied in industries to make smart predictions based on previous data collected and these IoT devices deployed are capable of making accurate decisions as compared to humans by collecting and communicating real time data.

Industrial monitoring is an important part of industries nowadays. This monitoring device will monitor the industrial equipment and it is also used to know the working status of the machines and industrial components. There are many methods to monitor the industrial process but the most efficient and most used is "Internet of Things".

Release of any dangerous gas or liquid can be one of the reasons for industrial accidents. The release of these gas or liquid may lead to the development of a direct or indirect gas cloud (vapour cloud). The direct gas cloud may develop after the release of gases from the chamber or a tank. The indirect

Revised Version Manuscript Received on 10 September, 2019.

D.UshaNandini, Sathyabama Institute of Science and Technology, Chennai, Tamilnadu, India.

S.Prince Mary, Sathyabama Institute of Science and Technology, Chennai, Tamilnadu, India.

R.Sathyabama Krishna, Sathyabama Institute of Science and Technology, Chennai, Tamilnadu, India

B.Bharathi, Sathyabama Institute of Science and Technology, Chennai, Tamilnadu, India

V.MariaAnu, Sathyabama Institute of Science and Technology, Chennai, Tamilnadu, India gas cloud may be a consequence of leakage of liquid substance that forms a pool. If a flammable gas or vapour gets into the air and there is an ignition source present nearby, then there is a high chance of fire explosion. Thus, we need to keep monitoring the industrial space and keep all the precautionary actions ready in case of any misfortune.

\section{RELATED WORK}

Industrial IoT is an enduring and an active topic for all the scientists and engineers. It has solved many issues in the industries and also introduces new technologies every year to the industries. In this section, we discuss the existing environmental monitoring and management issues and previous systems.

\section{A. Gas detection system}

Gas detection devices have been used by various industries for a long time. Gas detection systems were introduced first for the mining industry this was used to identify any particular place in the mining area where it is dangerous for human beings to enter as some places tend to have high level of harmful gases which was impossible for humans to identify without entering. So this device was introduced in the market for the safety of miners. As the technology progressed a wide range of applications were developed for gas detectors like in industrial activities, refineries, manufacturing plants, transportation, even for commercial vehicles and houses [2,9].

\section{B. Pollution monitoring system}

Pollution monitoring is an exercise to measure the levels of air pollution in an area. This helps in understanding the status of the air quality we breathe. Creating such systems with the help of IoT helps us to not only to analyse the quality of air but also to store these results for future use. Policy makers have often cited these systems as an integral part of a pollution control strategy. These systems were also introduced for vehicles to monitor pollution caused by the vehicles. To detect pollution these systems often use specific gas sensors like NDIR sensors (carbon dioxide and carbon monoxide), NOx sensor (nitrogen oxide), ESP8266 (Dust sensor), etc $[3,8]$.

\section{Environmental Monitoring}

Environmental observance could be a method that's performed to characterize and monitor the standard of the setting. These systems were introduced to reduce human

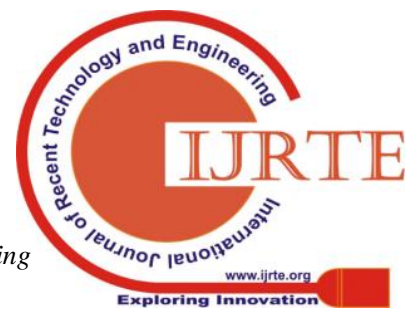


effort to gather information about the environment and also to assist human beings in many situations or circumstances in which a risk is involved. There are many applications of these systems like for air quality monitoring, soil monitoring, water monitoring, etc. As these systems are used to monitor large area and collect a lot data it is important to manage these data and store them [4].

\section{Equipment Monitoring}

Every year a lot of money is spent on maintenance of the equipment used in the manufacturing industries and often some loss is occurred because of failure of the equipment. But in the recent years, equipment monitoring is being used and using equipment monitoring the failure of the equipment can be predicted using the previous stored information. This system of monitoring equipment is based on IoT. These systems monitor the equipment frequently so that their performance is noted in every situation and if any unusual thing is identified then the admin is requested to review the equipment so that any future damage to the equipment is prevented [5-7].

\section{PROPOSED SYSTEM}

The proposed system uses IoT to monitor the environment of the industrial space and stores the data when a change is detected. The system is integrated with MQ2 gas sensor which is capable of detecting gases like Carbon monoxide, Hydrogen, Methane, Propane, LPG, Smoke, Alcohol, etc. When these gases are present around this vehicle the sensor detects and it notifies to the user and simultaneously uploads the data to the cloud using the IoT module. When a gas sensor detects the presence of a gas the data is sent to the microcontroller which checks the safety or permissible limits of the gas and a notification is sent to the user through GSM device. The module is not a fixed system. It can be maneuvered by humans through the Bluetooth device and smartphone. Also it can be made to move like an automated vehicle for which the Ultrasonic sensor is being used. Hence it also becomes a obstacle avoiding system.

\section{IMPLEMENTATION}

\section{A. Architecture of the system}

The block diagram shown in Fig 1 is the system architecture of the proposed system. This monitoring system is a movable system that has a robot mechanism that acts as the body of the system that will carry all the other components and the robot mechanism will have four wheels like that of a car and two DC motors are used to run the vehicle. As a power supply, a battery is used. The Arduino Mega is used which is a microcontroller that will be connected to each component and it will control all the sensors.

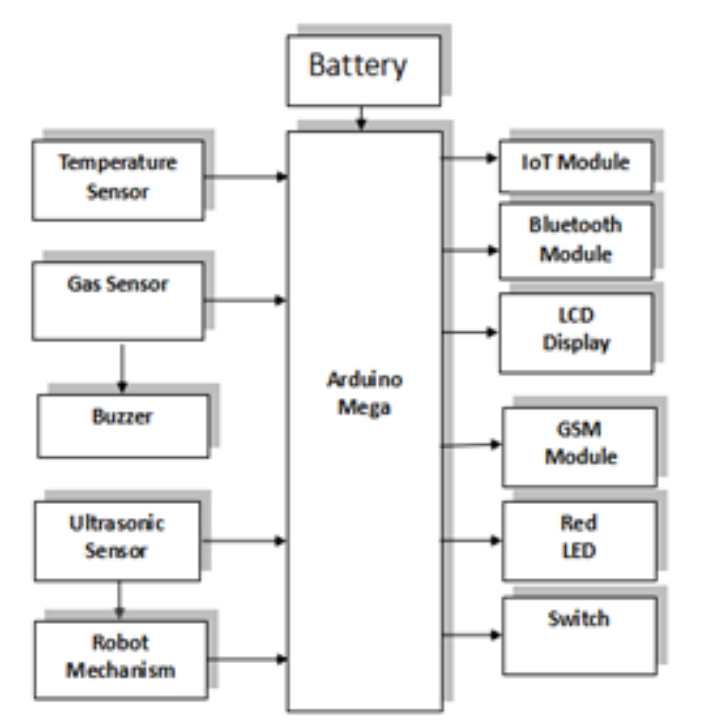

Fig 1. Architecture of the proposed system

An ultrasonic sensor is placed in front of the vehicle so that it will detect any object within distance of $20 \mathrm{~cm}$ and if any obstacle is detected then the vehicle will change its direction accordingly. This makes the vehicle less dependable on humans. The other components used are LCD display to show the real time data, an IoT module to store and upload the real time data in cloud, GSM module is used to send an alert message to the admin when a change in atmosphere is detected. A gas sensor and a temperature sensor is used to monitor the environment.

The whole architecture of the system is divided into three modules.

\section{Obstacle avoidance vehicle}

The first module is an obstacle avoidance vehicle that is used to detect any obstacle in the path of the vehicle. Using ultrasonic sensor the obstacle is detected and the path of the vehicle is changed automatically without any human interaction. This module is proposed to make the whole system movable. By making the whole monitoring system movable the application of the system is increased because this monitoring system can be used in an area where humans can't enter or humans cannot be present there for a long time. The architecture of the first module is given in Fig 2. The first module act as a base for the next two modules.

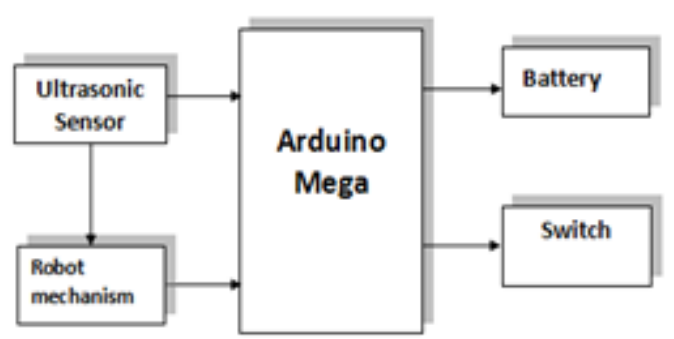

Fig 2. Architecture of obstacle avoidance system 


\section{Gas Detection System}

The second module is a gas detection system that is used to monitor the industrial space. This module allows the admin to monitor the industrial area without being present at the site. This system consists of three components apart from the components used in first module. Gas sensor is used to check the presence of any gas of any chemical. More than one gas sensors can be used together. MQ2 or MQ7 can be used to detect gases. Along with sensors a LCD display is used to display the gas detection status whether any gas is detected or not. The LCD also displays the reading of the ultrasonic sensor. In this module, Bluetooth HC-05 module is used to control the system wireless. The Bluetooth module allows the vehicle to move without direct assistance from humans. The Bluetooth module can be connected to an android app that will have voice commands or button commands. Using these commands the vehicle can be run. The purpose of using Bluetooth component is to control the vehicle wireless so that the vehicle can be controlled with comparatively far distance.

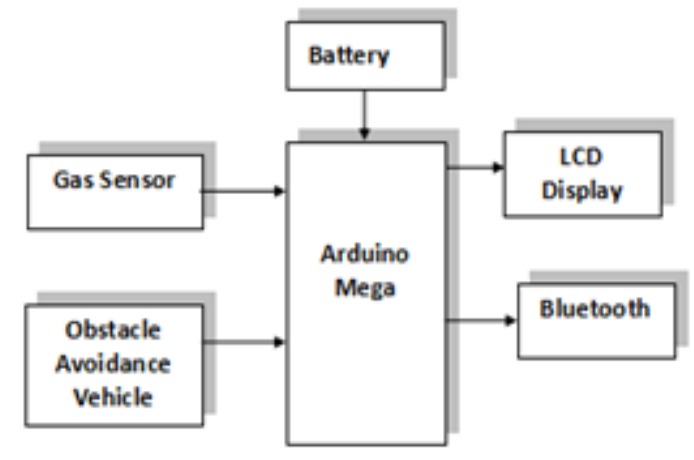

Fig 3. Architecture of Gas Detection System

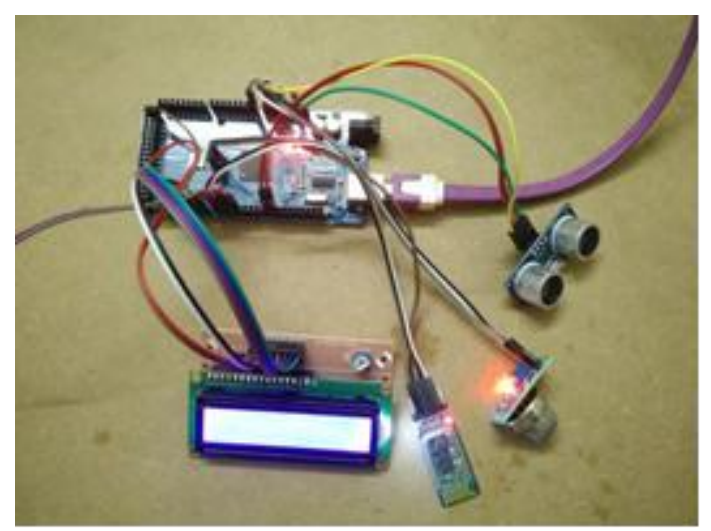

Fig 4. Gas detection system integrated with ultrasonic sensor and LCD panel.

\section{Communication Module}

The third module is the final module, which is proposed to perform the task of communication. The data that is collected by the sensor can be utilized for future predictions and can be used to find some kind of pattern in the results obtained by the sensors.

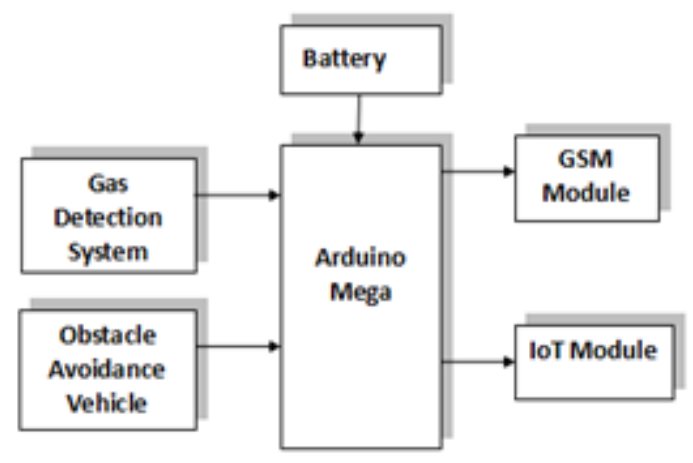

Fig 5. Architecture of Communication module

The information collected can be stored using the IoT module and using GSM module the information can be sent as an alert message to the admin. A Buzzer or a LED can also be used to notify the admin about the change in environment.

\section{RESULTS}

After implementation of each modules the following results for monitoring industrial area are obtained.

The first module, "Obstacle avoidance car" takes the input from ultrasonic sensor and finds the distance between the vehicle and the nearest object in front of the car. If the distance is less than $20 \mathrm{~cm}$ then the car moves its path automatically.

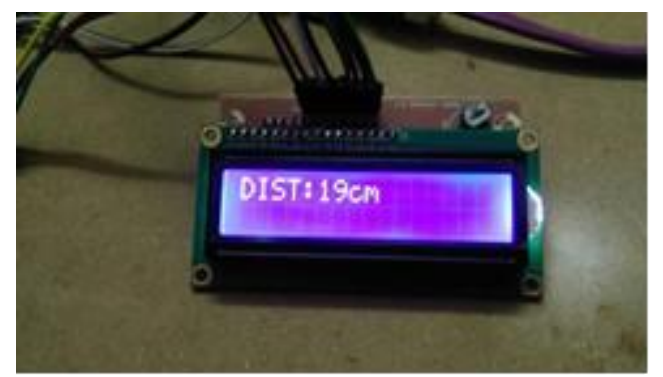

Fig 6. Distance calculated by ultrasonic sensor

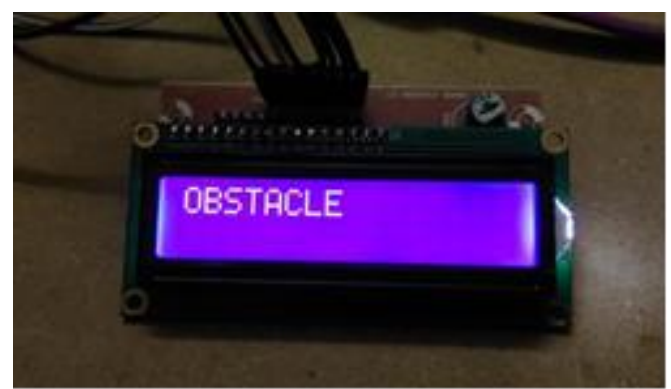

Fig 7. Obstacle detected 
The second module, "Gas detection system" takes the air components as the input through the gas sensor and if any gas is identified in the air then the message is displayed.

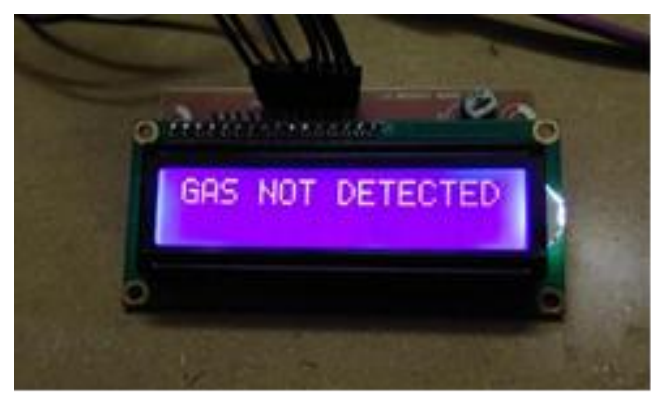

Fig 8. Gas not detected by the sensor

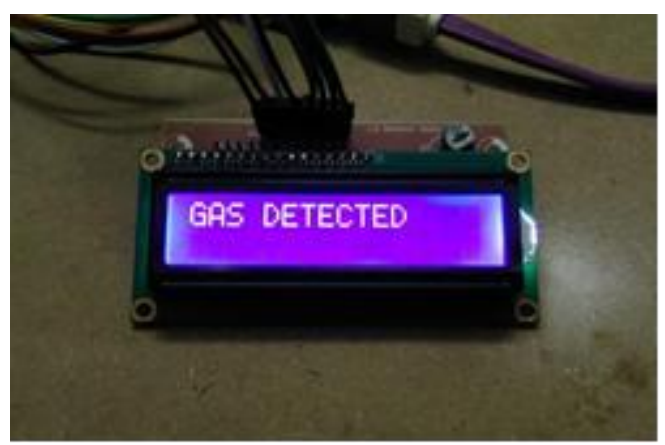

Fig 9. Gas detected by the sensor

The third module, "Communication module" is used to communicate any output generated by the two modules to the admin or user. Using GSM module, the admin is alerted with a SMS and through IoT module these data are stored online for future use.

\section{CONCLUSION}

This paper introduces an IoT based system for monitoring industrial spaces. This vehicle system is useful for the industries which has a gas chamber or space where there is possibility of a gas leak. Different industries can use respective gas sensor as required. This system is movable system unlike the existing systems.

\section{REFERENCES}

1. "Internet Of Things" from Wikipedia, "https://en.wikipedia.org/wiki/Internet_of_things".

2. Kumar Keshamoni, SabbaniHemanth, "Smart Gas Level Monitoring, Booking \& Gas Leakage Detector over IoT", 2017 IEEE 7th International Advance Computing Conference.

3. Chen Xiaojun, Liu Xianpeng, XuPeng, "IOT- Based Air Pollution Monitoring and Forecasting System", International Conference on Computer and Computational Sciences (ICCCS) 2015.

4. Shifeng Fang, Li Da Xu, Yunqiang Zhu, JiaerhengAhati, Huan Pei, Jianwu Yan, and Zhihui Liu, "An Integrated System for Regional EnvironmentalMonitoring and Management Based onInternet of Things", IEEE TransactionsonIndustrialInformatics, Vol. 10, No. 2, MAY 2014

5. SuratsavadeeKoonlaboonKorkue, Wei-Jen Lee, "Wireless Sensor network for permance monitoring of electricalmachines,"North American Power Symposium(NAPS), pp. 1-5, Octobor 2009.

6. Anu, V. Maria, GS Anandha Mala, and K. Mathi.
"Comparison of RFID data processing using dimensionality reduction techniques." In 2014 International Conference on Control, Instrumentation, Communication and Computational Technologies (ICCICCT), pp. 265-268. IEEE, 2014.

7. Gladence, L. Mary, M. Karthi, and V. Maria Anu. "A statistical comparison of logistic regression and different Bayes classification methods for machine learning." ARPN Journal of Engineering and Applied Sciences 10, no. 14 (2015): 5947-5953.

8. L.MaryGladence, "Detection of Contiguous Pattern with Pattern Shift String Matcher", Research Journal of Pharmaceutical, Biological and Chemical Sciences ISSN 0975-8585 Volume 8,Issue2,2017(March-April) Page. No. $2449-2456$

9. Maria Anu, V., GS Anandha Mala, and K. Mathi. "An Overview of RFID Data Processing Techniques." International Journal of Applied Engineering Research 9, no. 21 (2014): 8603-8612.

10. Mary Gladence L, Hari Haran S, Gopinath V, ShanmugaPriya.S,"Home and Office Automation System using Human Activity Recognition" in international conference on Communicatio and Signal Processing(ICCSP-2017)6th to 8th April 2017 in Adiparasakthi Engineering College.DOI 10.1109/ICCSP.2017.8286463. 